\title{
An in vitro study showing the three-dimensional microenvironment influence over the behavior of head and neck squamous cell carcinoma
}

\author{
Fernanda Salgueiredo-Giudice ${ }^{1}$, Aline Corrêa-Abrahão ${ }^{1}$, Felipe Fornias-Sperandio ${ }^{1}$, Aluana-Maria da-Cos- \\ ta-Dal-Vechio ${ }^{1}$, Décio dos-Santos-Pinto-Junior ${ }^{2}$
}

${ }^{1}$ DDS, MSc, PhD student, Department of Oral Pathology, School of Dentistry, University of São Paulo, São Paulo, SP, Brazil

${ }^{2}$ DDS, MSc, PhD, Professor, Department of Oral Pathology, School of Dentistry, University of São Paulo, São Paulo, SP, Brazil

Correspondence:

Department of Oral Pathology, School of Dentistry

University of São Paulo, Brazil

Av. Professor Lineu Prestes

2227 São Paulo, SP, Brazil CEP: 05508-000

fernandagiudice@usp.br

\begin{abstract}
Salgueiredo-Giudice F, Corrêa-Abrahão A, Fornias-Sperandio F, da-Costa-Dal-Vechio AM, dos-Santos-Pinto-Junior D. An in vitro study showing the three-dimensional microenvironment influence over the behavior of head and neck squamous cell carcinoma. Med Oral Patol Oral Cir Bucal. 2012 May 1;17 (3):e377-82.

http://www.medicinaoral.com/medoralfree01/v17i3/medoralv17i3p377.pdf
\end{abstract}

Received: $11 / 02 / 2011$ Accepted: 17/04/2011

\begin{abstract}
Objectives: The Head and Neck Squamous Cell Carcinoma (HNSCC) ranks sixth worldwide. The mechanisms of growth, invasion and metastasis of this pathology are extensively studied and generally related to specific variations in signaling pathways like the PI3K-Akt; however most of these competent studies have been performed bidimensionally, which may hide important questions. This study sought to analyze the influence of the microenvironment upon the behavior of HNSCC.

Study Design: The status of pAkt, NF-кB and Cyclin D1 proteins was accessed through immunofluorescence and western blot methods in HNSCC cell lines originating from tongue, pharynx and metastatic lymph node when submitted to a three-dimensional culture model utilizing a matrix system. A bidimensional culture model (monolayer) was used as control.

Results: The HNSCC cell lines cultured three-dimensionally exhibited a growth pattern characterized by small isolated islands, different from the control group. When the three-dimensional model was applied, two of the studied cell lines showed the same expression pattern as the bidimensional model regarding nuclear or cytoplasmatic localization, as well as reduction of all protein levels; however, the cell line originated from tongue, which specially has the epidermal growth factor receptor constitutively activated, demonstrated nuclear translocation of pAkt and also an increase in the levels of Cyclin D1.

Conclusions: The results suggest the influence of the microenvironment upon the behavior of HNSCC cells due to the changed expression of proteins related to tumor growth and cellular invasion. Furthermore, intrinsically genetic conditions also played important roles over the cells, despite the culture model employed.
\end{abstract}

Key words: Carcinoma, squamous cell, head and neck neoplasms, extracellular matrix, cell culture techniques, signal transduction. 


\section{Introduction}

As one of the most common cancers, Head and Neck Squamous Cell Carcinoma (HNSCC) is an aggressive malignancy that arises in the oral cavity, larynx and pharynx, resulting in 500.000 new cases per year in the world (1) and nearly 11.000 deaths in the United States alone (2).

In spite of a tendency in the early detection of HNSCC (3) and advances in cancer prevention or treatment, the five-year survival rate of a patient that receives the diagnosis of HNSCC remains low (approximately 50\%), which is even poorer than other cancers, such as colorectal, cervix and breast (2). Meanwhile, besides many of the most common risk factors involved in HNSCC development, such as alcohol and tobacco being well recognized, the complete knowledge of the mechanisms underlying the malignant progression of HNSCCs remains unclear (4).

In that way, many in vitro studies that focus on linking several signaling pathways with the cancer development and progression have been performed. Among the most studied proteins related to HNSCC invasion, cell proliferation and metastasis, pAkt, NF- $\kappa \mathrm{B}$ and Cyclin D1 can be cited (5). Nonetheless, most of the studies account for bidimensional models over plastic surfaces and, as cancer cells reside in vivo in a three-dimensional microenvironment, the monolayer model can hide important results $(6,7)$.

The PI3K-Akt signaling pathway, when activated, may prevent the cell death by inactivating pro-apoptotic factors and by activating transcription factors that upregulate anti-apoptotic genes, including the transcription factor NF- $\mathrm{kB}$, which is an essential component of intracellular regulatory circuitries of cell proliferation and survival (8).

In HNSCCs, the expression and activity of NF- $\kappa \mathrm{B}$ is often up-regulated, and its levels increase gradually from pre-malignant lesions to invasive cancer $(9,10)$, which suggests that NF- $\kappa B$ signaling pathway may play an important role at the early stages of carcinogenesis. Additionally, the crosstalk between NF- $\kappa$ B and STAT (signal transducer and activator of transcription) proteins leads to activation of growth promoting genes expression, such as c-myc and Cyclin D1 (11). Nevertheless, what leads to the persistent activation of NF- $\kappa \mathrm{B}$ in HNSCCs is still unclear.

The cell behavior is easily modulated by the extracellular microenvironment, which is exemplified by the highly variable changes in gene expression and, subsequently, phenotype of the cancer cells cultured in different conditions (12). Thus, this work sought to evaluate the expression of pAkt, NF- $\kappa \mathrm{B}$ and Cyclin D1 in HNSCC cell lines cultured with the traditional bidimensional method (monolayer) and with the threedimensional model. Recombinant extracellular matrix composed by some components of human extracellular matrix, such as laminin, type IV collagen, nidogen, entactin and heparan sulfate (13) was used to mimic threedimensional microenvironment.

\section{Material and Methods}

All experiments were performed in triplicate.

\section{Cell Lines and Culture Conditions}

Three HNSCC cell lines were used [HN6 (base of tongue), HN30 (pharynx), HN31 (lymphnode)] (14) and cultured in DMEM (Dulbelco's Modified Eagle's Medium) supplemented with $10 \%$ fetal bovine serum, $100 \mathrm{U} /$ $\mathrm{mL}$ penicillin and $100 \mu \mathrm{g} / \mathrm{mL}$ of streptomycin (SigmaAldrich, St. Louis, $\mathrm{MO}, \mathrm{USA}$ ) at $37^{\circ} \mathrm{C}$ in a humidified atmosphere and $5 \% \mathrm{CO}_{2}$.

Immunofluorescence Assay

The bidimensional experimental model consisted on seeding the HNSCC (HN6, HN30 and HN31) cell lines (2 $x$ 105) over coverslips for 72 hours; the three-dimensional model was obtained exactly as the first experimental group, but the cells were seeded with 1:3 Matrigel (BD Matrigel $^{\mathrm{TM}}$ Matrix, BD, Franklin Lakes, NJ, USA - product number 356231). The medium was discarded and the cells were then rinsed with phosphate buffer solution (1X PBS), fixed in cooled absolute methanol (6 minutes, $-20^{\circ} \mathrm{C}$ ), washed five times with $1 \mathrm{X}$ PBS and then blocked with $1 \%$ bovine serum albumin [(BSA) Sigma-Aldrich, St. Louis, MO, USA] for 30 minutes in a humidified chamber. Coverslips were incubated with anti-p-Akt1/2/3 (Ser 473) (1:50, Santa Cruz Biotechnology, Inc., Santa Cruz, CA, USA - sc7985-R), anti-NF-кB (1:50, Zymed, Invitrogen Corporation, Carlsbad, CA, USA - P65C), anti-Cyclin D1 (1:100, Santa Cruz Biotechnology, Inc., Santa Cruz, CA, USA - sc8396) diluted in blocking buffer for 90 minutes in a humidified chamber, washed five times with $1 \mathrm{X}$ PBS and incubated with a FITC conjugated antibody (Vector Laboratories, Ind., Burlingame, CA, USA) for 45 minutes in a dark humidified chamber. After washing five times with 1X PBS, the coverslips were mounted with mounting medium (Vectashield: DAPI, Vector Laboratories, Ind., Burlingame, CA, USA) and analyzed by fluorescence microscope (Axio Imager.A1 - Carl Zeiss).

Western blot Assay

For the bidimensional culture mode, the HNSCC cell lines (106) were seeded on $58 \mathrm{~cm}^{2}$ dishes and maintained at $37^{\circ} \mathrm{C}$ in a humidified atmosphere and $5 \% \mathrm{CO}_{2}$ for 72 hours, and washed three times with cold 1X PBS. Cells were lysed with lysis buffer at $4^{\circ} \mathrm{C}$ for 20 minutes. The cells were then scraped and the lysate was collected in a microfuge tube. The lysate was cleared by centrifugation at $13.000 \mathrm{rpm}$ for 20 minutes at $4^{\circ} \mathrm{C}$ and the supernatant (total cell lysate) was collected, being the protein concentration of all samples determined by using the BCA method (Pierce Biotechnology, Rockford, IL, USA). 
For the three-dimensional culture mode, the HNSCC cell lines (106) were re-suspended in 1:3 Matrigel and maintained at $37^{\circ} \mathrm{C}$ in a humidified atmosphere and $5 \% \mathrm{CO}_{2}$ for 72 hours. Later, 1X PBS was added and the sample was cleared by centrifugation at $4^{\circ} \mathrm{C}$ and the supernatant (Matrigel with 1X PBS) was discarded. The cell pellet was incubated in ice-cold lysis buffer [50 mmol/L Tris-HCl (pH7.4), $1 \mathrm{mmol} / \mathrm{L}$ EDTA, 150 $\mathrm{mmol} / \mathrm{L} \mathrm{NaCl}, 1 \%$ Triton X-100, $1 \%$ DOC, $0.1 \%$ SDS, with freshly added protease inhibitor cocktail (Chemicon, Sigma, St. Louis, MO, USA)] for 20 minutes at $4^{\circ} \mathrm{C}$ and clarified by centrifugation (13.000 rpm for $20 \mathrm{~min}-$ utes at $4^{\circ} \mathrm{C}$ ). Supernatant (total cell lysate) was collected and the protein concentration of all samples was also determined using the BCA method (Pierce Biotechnology, Rockford, IL, USA).

For western blotting analysis, $7 \mu \mathrm{g}$ of the protein from whole cell lysates was loaded onto each lane for gel electrophoresis and transferred to a PVDF membrane. Immunoblotting was performed using $0.1 \mathrm{M}$ Tris $(\mathrm{pH} 7.5)$, $0.9 \% \mathrm{NaCl}, 0.05 \%$ Tween- 20 with $5 \%$ nonfat dry milk or BSA (Sigma-Aldrich, St. Louis, MO, USA) as a blocking and antibody-dilution buffer, and working anti-sera for anti-p-Akt1/2/3 (Ser 473) (1:150, Santa Cruz Biotechnology, Inc., Santa Cruz, CA, USA - sc7985), anti-NF-кB (1:100, Zymed, Invitrogen Corporation, Carlsbad, CA, USA - P65C) and anti-Cyclin D1 (1:100, Santa Cruz Biotechnology, Inc., Santa Cruz, CA, USA - sc8396), followed by anti-mouse secondary antibody (IgG-HRP, Santa Cruz Biotechnology, Inc., Santa Cruz, CA, USA). Bound antibody was detected by a colorimetric method using an Opti $4 \mathrm{CN}$ kit (Bio-rad Laboratories, Hercules, CA, USA). Betaactin (1:6000, Sigma-Aldrich, St. Louis, MO, USA) was used to control the total volume of each sample.

The band intensity quantification was performed using Image J 1.39u software (ImageJ, U. S. National Institutes of Health, Bethesda, Maryland, USA).

The statistical analysis was carried out using SPSS 11.0 (SPSS Inc., Chicago, IL, USA). Student's t test (independent sample) was performed to compare the groups with a level of significance of $5 \%$.

\section{Results}

HNSCC cell lines growth patterns revealed by the immunofluorescence assay

The HNSCC cells cultured with the three-dimensional mode exhibited a growth pattern characterized by small isolated islands (Figs. 1B, 1D, 1F). The other experimental group though, showed an homogenous, sheetlike, growth appearance (Figs. 1A, 1C, 1E).

The three-dimensional model suggests pAkt's nuclear translocation.

The HN6 cell line, when cultured with the bidimensional mode, presented the pAkt expression mainly in the nuclei (Fig. 1A) and focally in the cytoplasm of the respective cells (Fig. 1A'). The expression was only nuclear when the three-dimensional culture mode was employed (Fig. 1B).

The pAkt expression was cytoplasmatic for the HN30 cell line when these cells were cultured with both the experimental methods.

The HN31 cell line demonstrated nuclear expression of pAkt when cultured bidimensionally, and the expression remained in the nuclei when the three-dimensional method was employed.

NF-kB and Cyclin D1 cellular localizations remain equal for both culture methods.

The expression of these proteins was maintained in both of the experimental groups (bidimensional and three-dimensional culture modes), remaining the NF-kB staining in the cytoplasm (Figures 1C, 1D) and the Cyclin D1 staining in the nuclei (Figures 1E, 1F) of all HNSCC cell lines.

Western blotting confirms the influence of the microenvironment over HNSCC cells.

The comparison of protein levels between the experimental groups is presented in table 1, (Fig. 2 and Fig. 3). The pAkt and NF-kB protein levels were reduced in the three-dimensional model. Cyclin D1 levels of HN30 and HN31 cell lines cultured three-dimensionally were decreased, but increased in the HN6 cell line.

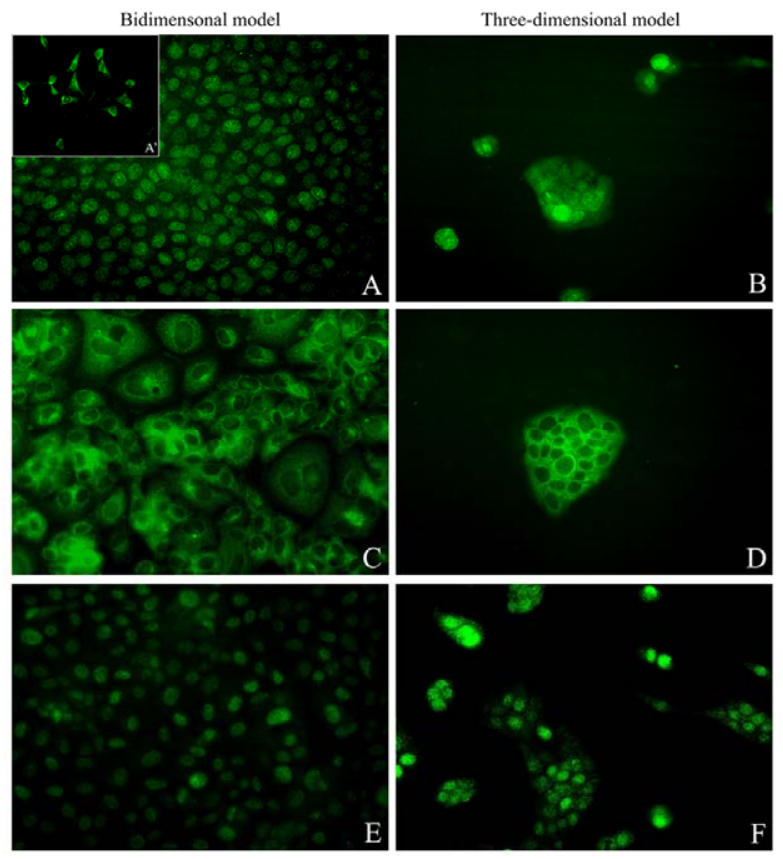

Fig. 1A. Nuclear pAkt expression in the HN6 cell line. A'-Focal cytoplasmatic staining of pAkt in the HN6 cells; B -Nuclear translocation of pAkt in the HN6 cells when cultured three-dimensionally;

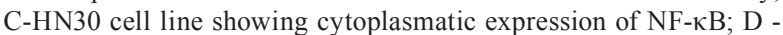
NF- $\kappa B$ expression remains in the cytoplasm of HN30 cells cultured with the matrix system; E - HN31 cell line showing nuclear expression of Cyclin D1; F - Cyclin D1 expression remains in the nuclei of the HN31 cells when cultured with the three-dimensional mode. 
Table 1. Respective $\mathrm{p}$ values obtained through the statistical comparison of bidimensional and three-dimensional Western blotting band quantifications.

\begin{tabular}{|c|c|c|c|}
\hline Cell lines & pAkt & NF-кB & Cyclin D1 \\
\hline HN6 & 0.0008 & 0.0002 & 0.0128 \\
\hline HN30 & 0.0731 & 0.0029 & 0.0002 \\
\hline HN31 & 0.0001 & 0.0003 & 0.0004 \\
\hline
\end{tabular}

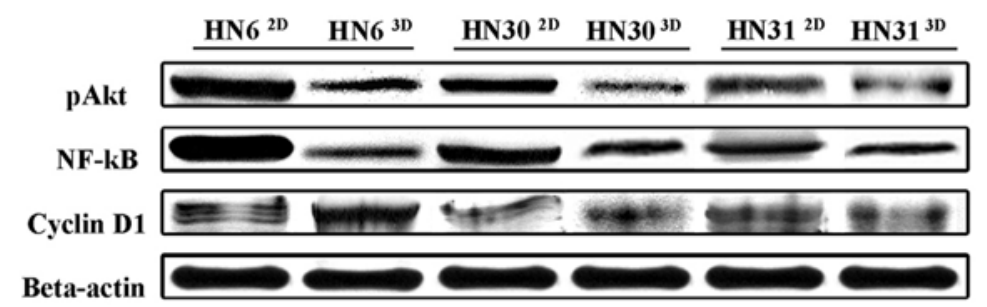

Fig. 2. Western blotting of pAkt, NF- $\kappa B$ and Cyclin D1 in the respective HNSCC cell lines, indicating protein expression levels. Beta-actin was used as control of the total volume of each sample. 2D - Bidimensional model; 3D - Three-dimensional model.

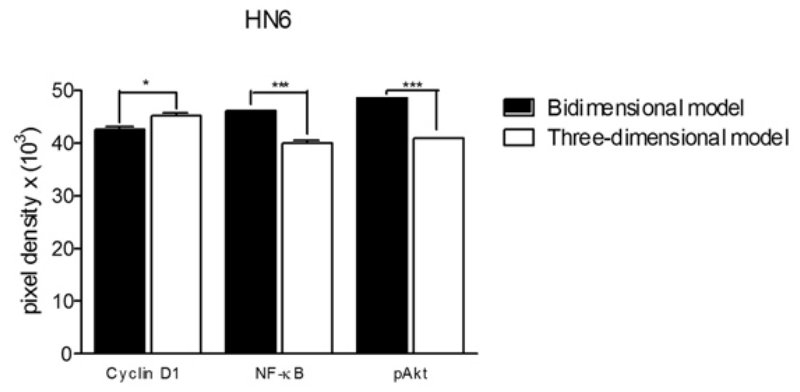

HN30

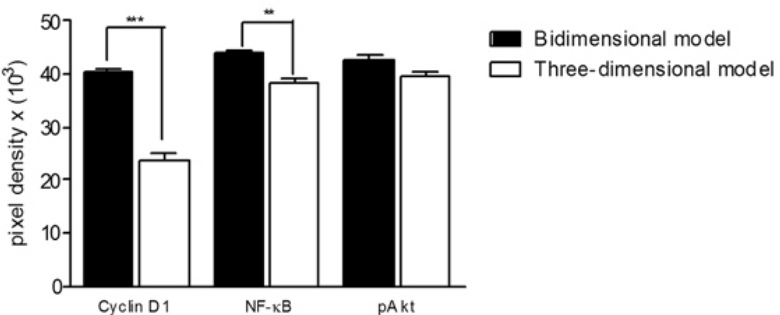

HN31

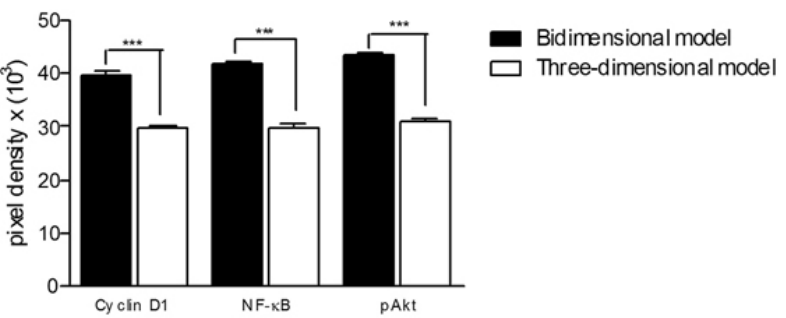

Fig. 3. Western blot quantified bands (Pixels X Band area); Mean values plus standard deviation. Statistically significant differences are represented by $*(\mathrm{p}<0.05) ; * *(\mathrm{p}<0.01) ; * *(\mathrm{p}<0.001)$. 


\section{Discussion}

The microenvironment plays an important role in the establishment and progression of carcinomas $(15,16)$, and although bidimensional studies consist on a convenient and rapid method of analysis, the real recreation of in vivo microenvironments is not feasible by these means. Accordingly, three-dimensional cell culture models may reproduce the physiological context regarding cell morphology, adhesion, proliferation and migration (17).

Three-dimensional culture models are currently being used to understand the signals that regulate the normal function of tissues and how they may change during pathological processes, such as cancer (18). In fact, the tumor growth is dictated not only by the interaction between tumor cells, but also by the interaction of these cells with their surrounding stroma (19).

Recent studies have demonstrated that different culture models may induce changes in the cells behavior. In that way, matrix systems have lead to distinct results if compared to bidimensional substrates (20). One of these matrix systems, Matrigel, is a commercially obtained extracellular matrix that can provide microenvironmental support, mimicking the natural architecture of the cells in vivo (21). In this study, the three-dimensional culture was characterized by a marked colony growth pattern that was different from that found with the bidimensional culture model.

The Akt protein may be activated in both cytoplasm and the nucleus (22), and while the pAkt's nuclear substrates are not yet totally described $(23,24)$, cytoplasmatic pAkt leads to NF- $\kappa \mathrm{B}$ activation (25). In this work, pAkt expression changed from the cytoplasm and nucleus to the nucleus solely, while the NF- $\kappa \mathrm{B}$ and Cyclin D1 cellular localization showed no differences. Although NF$\kappa \mathrm{B}$ was not found inside the nuclei of the cells, nuclear transcription factors remain inside the nucleus only for a short period of time, indicating that the transcripting role of NF- $\kappa \mathrm{B}$ in the HNSCC cells cannot be discarded (26). All protein levels diminished in the three-dimensional model, excluding the Cyclin D1 levels in the HN6 cell line, which showed an increase. The change from a bidimensional to a three-dimensional model leads to a decrease in pAkt and NF- $\kappa \mathrm{B}$ proteins levels, probably due to the cell adaptation period in Matrigel, which was characterized by the colony growth pattern (27) found herein. Additionally, Matrigel may influence cells behavior by reducing the stimulation of the PI3K-Akt sig-

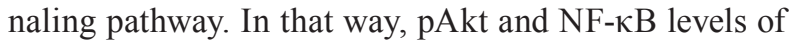
HN6, HN30 and HN31 cell lines cultured with Matrigel were decreased.

On the other hand, the HN6 cell line has the EGFR (epidermal growth factor receptor) constitutively activated (14), and thus showed an increase on the Cyclin D1 levels. Despite that the adaptation period probably forced the reduction of pAkt and NF- $\mathrm{BB}$ levels, the EGFR activation of other signaling pathways as STAT3 and Ras/ Map kinase (28) could be responsible for the Cyclin D1 increased levels in HN6 cells.

The present work showed interesting results about how the microenvironment may influence the behavior of carcinoma cells. The studied proteins, all of them related to carcinogenesis $(8,9,28)$, presented reduced levels in the cells cultured with Matrigel, which demonstrates how this matrix system can imply in different results concerning tumor growth and invasiveness. Moreover, intrinsic characteristics, such as a constitutively activated EGFR, also influenced the cell behavior, regardless the microenvironment.

When working with three-dimensional matrix systems, a simple fact that may alter gene expression as well as cellular behavior is the density of the scaffold gel (29). Denser matrix may favor the proliferation of breast epithelial cells (30). In that way, the Matrigel concentration utilized in the present study was 1:3. Further studies may clarify if different gel concentrations could influence the behavior of HNSCCs cell lines.

Finally, the results encountered herein allowed us to conclude that the microenvironment influenced the HNSCC cells behavior by changing the expression of proteins related to tumor growth and cellular invasion. Additionally, intrinsic genetic conditions may have also played important roles upon the cells, despite the culture model employed.

\section{References}

1. Parkin DM, Bray F, Ferlay J, Pisani P. Global cancer statistics, 2002. CA Cancer J Clin. 2005;55:74-108.

2. Jemal A, Siegel R, Ward E, Hao Y, Xu J, Murray T, et al. Cancer statistics, 2008. CA Cancer J Clin. 2008;58:71-96.

3. Marocchio LS, Lima J, Sperandio FF, Corrêa L, de Sousa SO. Oral squamous cell carcinoma: an analysis of 1,564 cases showing advances in early detection. J Oral Sci. 2010;52:267-73.

4. Bagan JV, Scully C. Recent advances in Oral Oncology 2007: epidemiology, aetiopathogenesis, diagnosis and prognostication. Oral Oncol. 2008;44:103-8.

5. Giudice FS, Dal Vechio AM, Abrahão AC, Sperandio FF, PintoJunior Ddos S. Different expression patterns of pAkt, NF- $\kappa B$ and cyclin D1 proteins during the invasion process of head and neck squamous cell carcinoma: an in vitro approach. J Oral Pathol Med. 2011;40:405-11.

6. Shekhar MP, Pauley R, Heppner G. Host microenvironment in breast cancer development: extracellular matrix-stromal cell contribution to neoplastic phenotype of epithelial cells in the breast. Breast Cancer Res. 2003;5:130-5.

7. Tlsty TD. Stromal cells can contribute oncogenic signals. Semin Cancer Biol. 2001;11:97-104.

8. Karin M, Greten FR. NF-kappaB: linking inflammation and immunity to cancer development and progression. Nat Rev Immunol. 2005;5:749-59.

9. Mishra A, Bharti AC, Varghese P, Saluja D, Das BC. Differential expression and activation of NF-kappaB family proteins during oral carcinogenesis: Role of high risk human papillomavirus infection. Int J Cancer. 2006;119:2840-50. 
10. Ondrey FG, Dong G, Sunwoo J, Chen Z, Wolf JS, Crowl-Bancroft $\mathrm{CV}$, et al. Constitutive activation of transcription factors NF-(kappa) B, AP-1, and NF-IL6 in human head and neck squamous cell carcinoma cell lines that express pro-inflammatory and pro-angiogenic cytokines. Mol Carcinog. 1999;26:119-29.

11. Reich NC, Liu L. Tracking STAT nuclear traffic. Nat Rev Immunol. 2006;6:602-12.

12. Wang R, Xu J, Juliette L, Castilleja A, Love J, Sung SY, et al. Three-dimensional co-culture models to study prostate cancer growth, progression, and metastasis to bone. Semin Cancer Biol. 2005; $15: 353-64$.

13. Xiang B, Muthuswamy SK. Using three-dimensional acinar structures for molecular and cell biological assays. Methods Enzymol. 2006;406:692-701.

14. Cardinali M, Pietraszkiewicz H, Ensley JF, Robbins KC. Tyrosine phosphorylation as a marker for aberrantly regulated growthpromoting pathways in cell lines derived from head and neck malignancies. Int J Cancer. 1995;61:98-103.

15. Cunha GR, Hayward SW, Wang YZ, Ricke WA. Role of the stromal microenvironment in carcinogenesis of the prostate. Int J Cancer. 2003;107:1-10.

16. Park CC, Bissell MJ, Barcellos-Hoff MH. The influence of the microenvironment on the malignant phenotype. Mol Med Today. 2000;6:324-9.

17. Griffith LG, Swartz MA. Capturing complex 3D tissue physiology in vitro. Nat Rev Mol Cell Biol. 2006;7:211-24.

18. Nelson CM, Bissell MJ. Modeling dynamic reciprocity: engineering three-dimensional culture models of breast architecture, function, and neoplastic transformation. Semin Cancer Biol. 2005;15:342-52.

19. Nielsen JD, Moeslund M, Wandall HH, Dabelsteen S. Influences of tumor stroma on the malignant phenotype. J Oral Pathol Med. 2008;37:412-6.

20. Cukierman E, Pankov R, Stevens DR, Yamada KM. Taking cellmatrix adhesions to the third dimension. Science. 2001;294:1708-12. 21. Sodunke TR, Turner KK, Caldwell SA, McBride KW, Reginato MJ, Noh HM. Micropatterns of Matrigel for three-dimensional epithelial cultures. Biomaterials. 2007;28:4006-16.

22. Wang R, Brattain MG. AKT can be activated in the nucleus. Cell Signal. 2006;18:1722-31.

23. Andjelković M, Alessi DR, Meier R, Fernandez A, Lamb NJ, Frech M, et al. Role of translocation in the activation and function of protein kinase B. J Biol Chem. 1997;272:31515-24.

24. Meier R, Alessi DR, Cron P, Andjelković M, Hemmings BA. Mitogenic activation, phosphorylation, and nuclear translocation of protein kinase Bbeta. J Biol Chem. 1997;272:30491-7.

25. Merkhofer EC, Cogswell P, Baldwin AS. Her2 activates NF-ka$\mathrm{ppaB}$ and induces invasion through the canonical pathway involving IKKalpha. Oncogene. 2010;29:1238-48.

26. Larue L, Bellacosa A. Epithelial-mesenchymal transition in development and cancer: role of phosphatidylinositol 3' kinase/AKT pathways. Oncogene. 2005;24:7443-54.

27 Delsanto PP, Condat CA, Pugno N, Gliozzi AS, Griffa M. A multilevel approach to cancer growth modeling. J Theor Biol. 2008;250:16-24.

28. Molinolo AA, Amornphimoltham P, Squarize CH, Castilho RM, Patel V, Gutkind JS. Dysregulated molecular networks in head and neck carcinogenesis. Oral Oncol. 2009;45:324-34.

29. Keely PJ, Fong AM, Zutter MM, Santoro SA. Alteration of collagen-dependent adhesion, motility, and morphogenesis by the expression of antisense alpha 2 integrin mRNA in mammary cells. J Cell Sci. 1995;108:595-607.

30. Boyd NF, Lockwood GA, Byng JW, Tritchler DL, Yaffe MJ. Mammographic densities and breast cancer risk. Cancer Epidemiol Biomarkers Prev. 1998;7:1133-44.

\section{Acknowledgement}

The authors were supported by predoctoral fellowship from CAPES, Brazil (Coordenação de Aperfeiçoamento de Pessoal de Nível Superior). 